\title{
Calibration method of 1D laser rangefinder for large-scale 3D measurement under harsh environment
}

\author{
Jiehu Kang ${ }^{1}$, Bin Wu ${ }^{1 \text { a) }}$, and Yuchan Wang ${ }^{2}$
}

\begin{abstract}
Vision measurement has encountered the bottlenecks in capturing and processing the images of laser spots under harsh working environment of strong sunshine and complex background. Breaking through the camera calibration, we propose that the corresponding relationship between pixel coordinates of laser spots, but not the camera, and measurement distances generated from laser rangefinder is directly calibrated under good working environment. The pixel coordinates of laser spots can be obtained from the calibration model and measurement distances during practical measurement. The imaging of laser spots by camera is no longer needed. Experimental results demonstrate that the measurement errors are in the millimeter level within $20 \mathrm{~m}$ distance outdoors.

Keywords: vision measurement, harsh working environment, laser rangefinder, calibration

Classification: Optical hardware
\end{abstract}

\section{Introduction}

The combination of laser rangefinder (LRF) and camera has been widely applied in various industrial measurements, such as the acquisition of urban models [1, 2, 3, $4]$, pedestrian detection [5, 6], robot motion planning [7] and smart car $[8,9]$. In these cases, it is necessary to know in advance the relative pose between camera and LRF for projecting the depth readings into the images.

The vast majority of applications are the fusion of $2 \mathrm{D}$ or 3D LRF and camera. Different extrinsic calibration techniques have been proposed in the literatures $[10,11$, 12, 13, 14, 15, 16]. Q. Zhang and R. Pless presented an extrinsic calibration method to estimate the orientation and position of a camera with respect to a 2D LRF for the robot [17]. The proposed method requires several poses of planar pattern, and a geometric constraint on the extrinsic camera parameters is imposed. F. Vasconcelos et al. proposed a minimal solution for the extrinsic calibration of a camera and a LRF [18]. The calibration is achieved by freely moving a checkerboard pattern in front of the two sensors to obtain the plane poses in camera coordinate system and depth readings in the LRF coordinate system.

Because only providing 1D length data, 1D LRF is seldom studied while it is very common in the engineering

${ }^{1}$ State Key Laboratory of Precision Measuring Technology and Instruments, Tianjin University, Tianjin 300072, China

${ }^{2}$ School of Optoelectronic Engineering, Chongqing University of Posts and Telecommunications, Chongqing 400065, China a)wubin@tju.edu.cn

DOI: 10.1587/elex.17.20200071

Received February 21, 2020

Accepted March 16, 2020

Publicized March 31, 2020

Copyedited April 25, 2020 and industrial applications. Recently the 1D LRF and camera are applied in the gun assembling smart targeting system. Z. Liu et al. proposed a method for calibrating the relative position and orientation of a $1 D$ LRF and single camera [19]. The proposed method requires a checkerboard to establish constraint equation, and the internal parameters of visible and infrared cameras must be calibrated.

According to the scale of measurement space, the 3D measurement tasks are roughly divided into large-scale measurement, regular scale measurement, and micro/nano scale measurement. This paper is mainly concerned with the large-scale measurement under harsh working environment. The measurement distance is from several meters to tens of meters and permissible measurement error is in the millimeter level. Concerning vision measurement, the harsh working environment is strong sunshine and complex background in this paper. Vision measurement has encountered the bottlenecks in capturing and processing the images of laser spots under harsh working environment. The laser spots generated from LRF cannot be captured by camera under strong sunshine and complex background. In other words, the camera cannot be employed for capturing the images of laser spots. The above proposed methods for calibrating the rigid-body transformation between LRF and camera are no longer applicable.

Aiming at solving this problem, we propose that the corresponding relationship between pixel coordinates of laser spots on the imaging plane and measurement distances generated from 1D LRF is directly calibrated under good working environment. In this proposed method, the pixel coordinate system, but not the camera coordinate system, is adopted during the calibration procedure. In other words, the pixel coordinates of laser spots and measurement distances are calibrated, but not the camera. The pixel coordinates of laser spots can be obtained from the calibration model and measurement distances during practical measurement. The imaging of laser spots by camera is no longer needed.

\section{Calibration principle}

The calibration principle is shown in Fig. 1. The coordinate system of camera is defined as $O_{c} x_{c} y_{c} z_{c}$. The coordinate system on the imaging plane is defined as $O x y$. In the coordinate system of $O_{c} x_{c} y_{c} z_{c}$, the starting point of measurement distance of LRF is defined as $P_{L 0}\left(x_{L 0}, y_{L 0}, z_{L 0}\right)$ and the direction vector of laser beam is defined as $\overrightarrow{v_{L}}=$ $(i, j, k)$. The laser beam of LRF can be expressed as 


$$
L(t)=P_{L 0}+t * \overrightarrow{v_{L}}
$$

where $t$ represents the length coefficient.

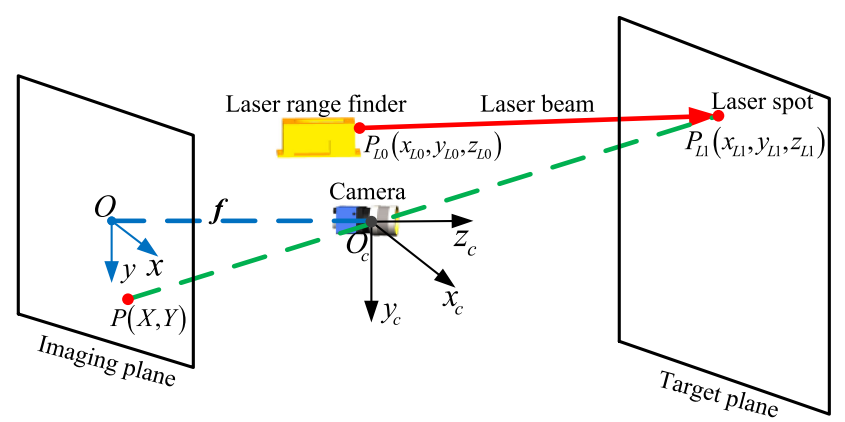

Fig. 1. The calibration principle of corresponding relationship between pixel coordinates of laser spots and measurement distances.

The laser spot on the target plane is defined as $P_{L 1}\left(x_{L 1}, y_{L 1}, z_{L 1}\right)$. The distance between $P_{L 0}$ and $P_{L 1}$ is defined as $L$, which can be obtained from LRF. The 3D coordinate of laser spot on the target plane can be described as

$$
\left[\begin{array}{c}
x_{L 1} \\
y_{L 1} \\
z_{L 1}
\end{array}\right]=\left[\begin{array}{c}
x_{L 0}+L * i \\
y_{L 0}+L * j \\
z_{L 0}+L * k
\end{array}\right]
$$

The projection point of laser spot from the target plane onto the imaging plane is defined as $P(X, Y)$. Based on the perspective projection model $[20,21]$, the $2 \mathrm{D}$ coordinate of projection point on the imaging plane in the coordinate system of $O x y$ can be expressed as

$$
\left[\begin{array}{l}
X \\
Y
\end{array}\right]=f\left[\begin{array}{l}
\frac{x_{L 1}}{z_{L 1}} \\
\frac{y_{L 1}}{z_{L 1}}
\end{array}\right]
$$

where $f$ represents the focal length of camera. Combining Eqs. (2) and (3), the corresponding relationship between pixel coordinates of laser spots and measurement distances can be expressed as

$$
\left[\begin{array}{c}
X \\
Y
\end{array}\right]=f\left[\begin{array}{c}
\frac{x_{L 0}+L * i}{z_{L 0}+L * k} \\
\frac{y_{L 0}+L * j}{z_{L 0}+L * k}
\end{array}\right]
$$

In Eq. (4), $(X, Y), L$ and $f$ are known. The 2D coordinate of projection point $(X, Y)$ can be obtained by image processing. The unknown values are the parameters of laser beam, including the starting point $P_{L 0}\left(x_{L 0}, y_{L 0}, z_{L 0}\right)$ and the direction vector $\overrightarrow{v_{L}}=(i, j, k)$. The target plane is freely moved in front of LRF and camera more than three positions. And more than three sets of $(X, Y)$ and $L$ are obtained. The unknown parameters are derived by least square method and substituted into Eq. (4). The calibration model of pixel coordinates of laser spots on the imaging plane and measurement distances generated from LRF is obtained. From Eq. (4), the changing trend of pixel coordinates of laser spots is a part of hyperbola with the increase of measurement distance. To further optimize the corresponding relationship, the relative installation pose of LRF and camera are taken into account. To simplify the analysis, the Y pixel coordinate is taken as an example. The equation of Y pixel coordinate is expanded and formulated as

$$
Y=\frac{j}{k} * f+\frac{y_{L 0}-z_{L 0} * \frac{j}{k}}{z_{L 0}+L * k} * f
$$

Assume that the direction vector of laser beam is approximatively parallel to the z-axis of $O_{c} x_{c} y_{c} z_{c}$ and the starting point of LRF is approximatively coincided with the origin of $O_{c} x_{c} y_{c} z_{c}$. And the measurement distance $L$ is much greater than $y_{L 0}$ and $z_{L 0}$. The geometric inequality is obtained

$$
y_{L 0}-z_{L 0} * \frac{j}{k} \ll z_{L 0}+L * k
$$

The Y pixel coordinate can be expressed as

$$
Y=\frac{j}{k} * f
$$

Thus the changing trend of pixel coordinates of laser spots is converted from a part of hyperbola to a line segment.

The above proposed method is applied to articulated laser vision sensor. The novel sensor mainly consists of two rotary tables, one LRF and one camera. The axes of sensor represent a non-orthogonal shafting architecture $[22,23]$. The requirements of structural design, processing technology, assembling, and maintain are greatly lowered. The LRF and camera are mounted on the same rotary table. In actual assembly of articulated laser vision sensor, the direction vector of laser beam can be approximatively parallel to the z-axis of $O_{c} x_{c} y_{c} z_{c}$. However, the starting point of LRF cannot be approximatively coincided with the origin. There is a certain distance in the $\mathrm{x}$-axis, which cannot be ignored. Thus the changing trend of $\mathrm{X}$ pixel coordinates is a part of hyperbola and the changing trend of $\mathrm{Y}$ pixel coordinates is a line segment.

\section{Experiments}

The calibration setup is shown in Fig. 2. An optical calibration plate is used to receive the laser spots and defined as the target plane. The target plane is freely moved in front of LRF and camera.

The flow chart of calibration procedure is shown in Fig. 3. The calibration distance is approximately from $5 \mathrm{~m}$ to $20 \mathrm{~m}$.

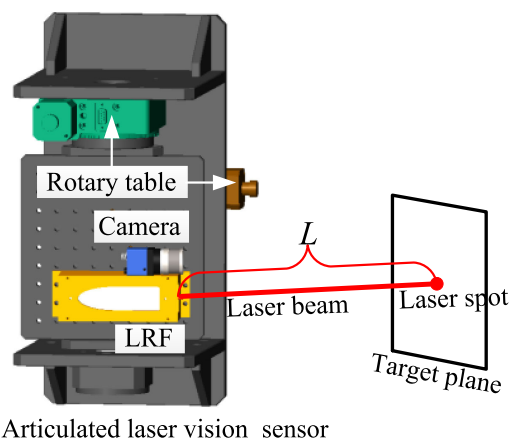

Fig. 2. Calibration setup of pixel coordinates and measurement distances. 


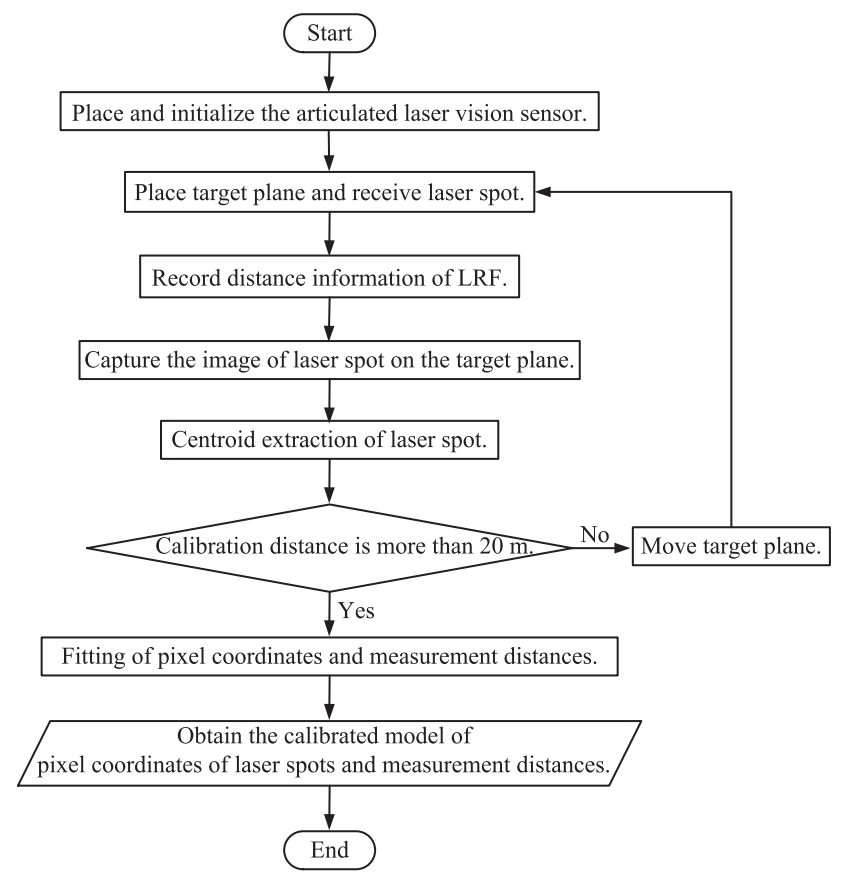

Fig. 3. The flow chart of calibrating the pixel coordinates and measurement distances.

The calibration experiment is designed under good working environment. The target plane is placed in 27 positions and 27 images of laser spots are captured. 27 sets of pixel coordinates of laser spots on the imaging plane $[24,25,26]$ and measurement distances generated from LRF are obtained. The corresponding relationships are fitted by Levenberg-Marquardt method [27, 28, 29, 30]. The fitting results are shown in Fig. 4, Fig. 5, Table I and Table II.

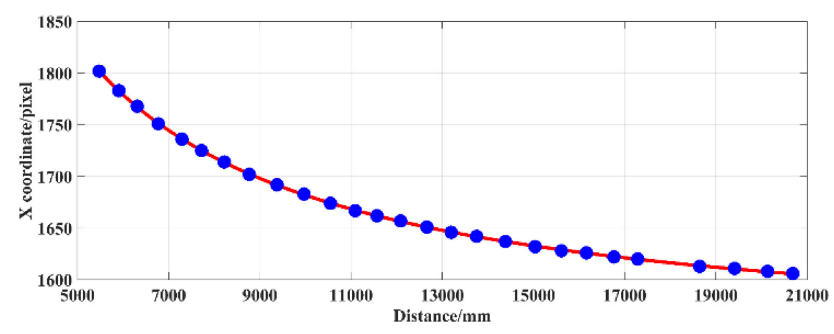

Fig. 4. The $\mathrm{X}$ pixel coordinates and measurement distances.

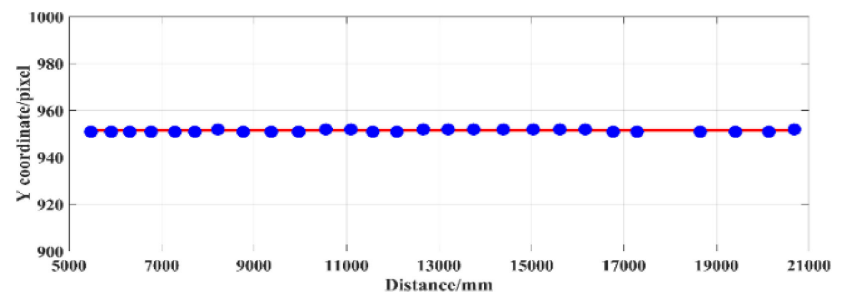

Fig. 5. The Y pixel coordinates and measurement distances.

The pixel coordinates of laser spots on the imaging plane can be obtained from the calibration model and measurement distances during practical measurement. Fortunately, the imaging and extraction of laser spots are no longer needed under harsh working environment. The
Table I. The fitting results of X pixel coordinates. Unit: pixel

\begin{tabular}{c|c|c}
\hline Max deviation & Min deviation & RMSE \\
\hline 0.94 & 0.01 & 0.51 \\
\hline
\end{tabular}

Table II. The fitting results of Y pixel coordinates. Unit: pixel

\begin{tabular}{c|c|c}
\hline Max deviation & Min deviation & RMSE \\
\hline 0.50 & 0.50 & 0.50 \\
\hline
\end{tabular}

practical measurement experiment is performed under strong sunshine and complex background outdoors. The flow chart of large-scale 3D measurement is shown in Fig. 6. 39 measured points are placed in different positions, as shown in Fig. 7. The 3D coordinates of measured points are measured by laser tracker, which are defined as reference values. The $3 \mathrm{D}$ coordinates of measured points are measured by articulated laser vision sensor, which are defined as measured values. The deviations of measured values and reference values are shown in Fig. 8, Fig. 9, Table III and Table IV.

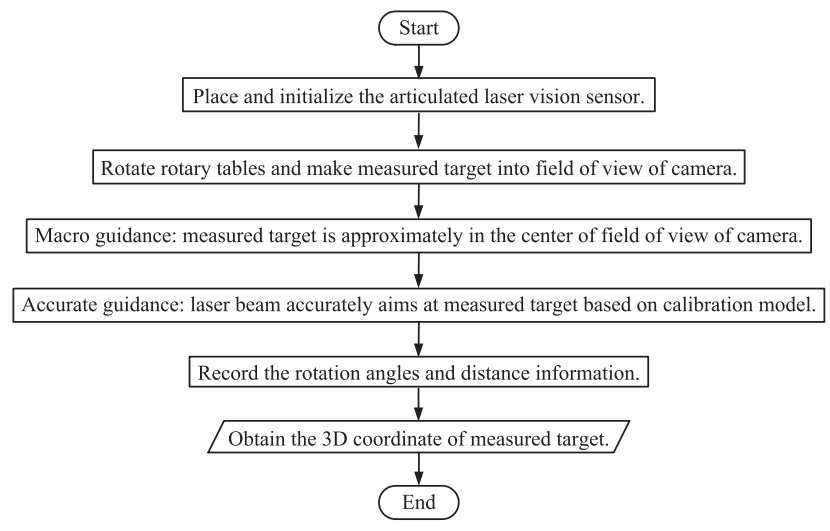

Fig. 6. The flow chart of large-scale 3D measurement of articulated laser vision sensor.

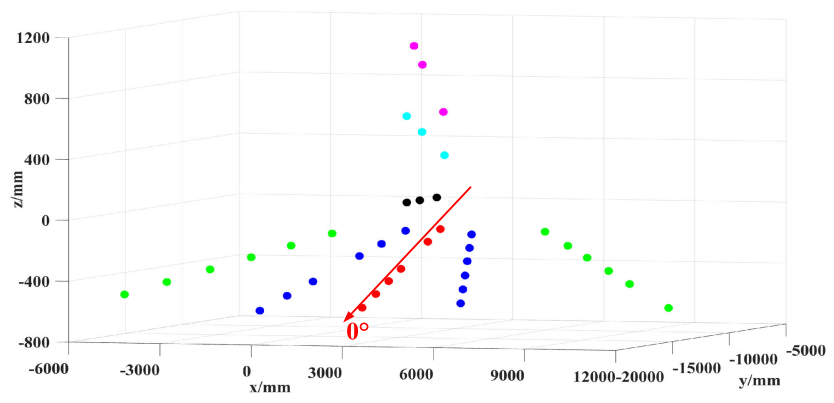

Fig. 7. The measured points in different distribution positions.

From the Fig. 7, Fig. 8, Fig. 9, Table III and Table IV, the experimental results are obtained as follows. First, the measurement errors are in the millimeter level within $20 \mathrm{~m}$ measurement distance. Second, the measurement error evidently increases with the increase of measurement distance. Third, the measurement error increases with the increase of the horizontal and vertical divergence angles, whose zero reference is shown in Fig. 7. 


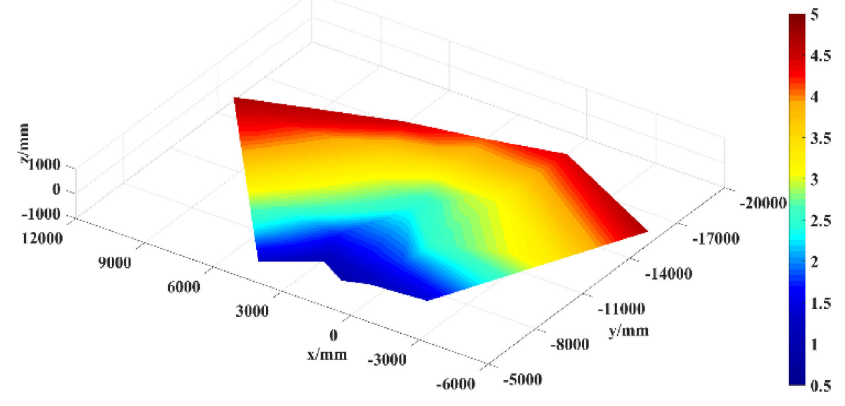

Fig. 8. The deviations of measured values and reference values in the horizontal direction.

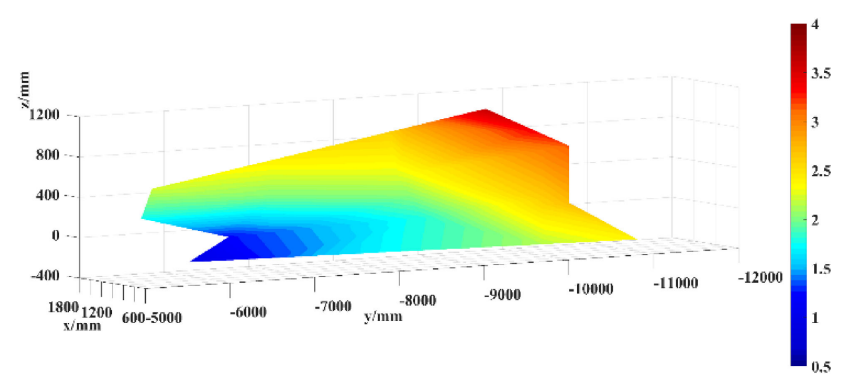

Fig. 9. The deviations of measured values and reference values in the vertical direction.

Table III. Comparison between measured values and reference values in the horizontal direction. Unit: $\mathrm{mm}$

\begin{tabular}{c|c|c|c}
\hline Max deviation & Min deviation & Average & Std. deviation \\
\hline 4.72 & 0.94 & 2.85 & 1.15 \\
\hline
\end{tabular}

Table IV. Comparison between measured values and reference values in the vertical direction. Unit: $\mathrm{mm}$

\begin{tabular}{c|c|c|c}
\hline Max deviation & Min deviation & Average & Std. deviation \\
\hline 3.62 & 0.94 & 2.19 & 0.78 \\
\hline
\end{tabular}

\section{Conclusion}

In conclusion, we propose an effective calibration method of 1D LRF for large-scale 3D measurement under harsh working environment of strong sunshine and complex background. The corresponding relationship between pixel coordinates of laser spots on the imaging plane and measurement distances generated from LRF is directly calibrated under good working environment. The camera calibration is no longer needed. During practical measurement, the pixel coordinates of laser spots can be obtained from the calibration model instead of the imaging of laser spots by camera. This proposed method breaks through the bottlenecks in capturing and processing the images of laser spots under harsh working environment. We experimentally demonstrate that the errors are in the millimeter level within $20 \mathrm{~m}$ measurement distance. With the advantages of cost-effective, good operability and high efficiency, this proposal offers a feasible solution to achieve large-scale 3D measurement under harsh working environment. This proposed method has been successfully applied in 3D shape detection of aircraft, which are outdoors.

\section{Acknowledgments}

This work is supported by the National Natural Science Foundation of China under Grant (No. 61771336, 61804020), the Natural Science Foundation of Tianjin in China under Grant No. 18JCZDJC38600, the Natural Science Foundation Project of Chongqing Grant No. cstc2019jcyj-msxmX0124.

\section{References}

[1] J. L. Blanco, et al.: "A collection of outdoor robotic datasets with centimeter-accuracy ground truth," Auton. Robots 27 (2009) 327 (DOI: 10.1007/s10514-009-9138-7).

[2] C. Früh and A. Zakhor: "An automated method for large-scale, ground-based city model acquisition," Int. J. Comput. Vis. 60 (2004) 5 (DOI: 10.1023/B:VISI.0000027787.82851.b6).

[3] F. T. Ramos, et al.: "Recognising and modelling landmarks to close loops in outdoor slam," IEEE International Conf. Robotics and Automation (2007) 2036 (DOI: 10.1109/ROBOT.2007.363621).

[4] B. Douillard, et al.: "Classification and semantic mapping of urban environments," Int. J. Robot. Res. 30 (2011) 5 (DOI: 10.1177/ 0278364910373409).

[5] C. Premebida, et al.: "Lidar and vision-based pedestrian detection system,” J. Field Robot. 26 (2009) 696 (DOI: 10.1002/rob.20312).

[6] C. Premebida, et al.: "Fusing lidar, camera and semantic information: A context-based approach for pedestrian detection," Int. J. Robot. Res. 32 (2013) 371 (DOI: 10.1177/0278364912470012).

[7] H. Baltzakis, et al.: "Fusion of laser and visual data for robot motion planning and collision avoidance," Mach. Vis. Appl. 15 (2003) 92 (DOI: 10.1007/s00138-003-0133-2).

[8] G. Kim, et al.: "Vision-based vehicle detection and inter-vehicle distance estimation for driver alarm system," Opt. Rev. 19 (2012) 388 (DOI: 10.1007/s10043-012-0063-1).

[9] L. Chen, et al:: "Design and implementation of the tracing smart car system based on laser sensors," International Conference on Computer Design and Applications 2 (2010) 598 (DOI: 10.1109/ ICCDA.2010.5541296).

[10] G. H. Li, et al.: "An algorithm for extrinsic parameters calibration of a camera and a laser range finder using line features," IEEE International Conference on Intelligent Robots and Systems (2007) 3854 (DOI: 10.1109/IROS.2007.4399041).

[11] L. X. Meng, et al.: "Extrinsic calibration of a camera with dual 2D laser range sensors for a mobile robot," IEEE International Symposium on Intelligent Control (2010) 813 (DOI: 10.1109/ISIC. 2010.5612903).

[12] R. Gomez-Ojeda, et al.: "Extrinsic calibration of a 2D laserrangefinder and a camera based on scene corners," IEEE International Conference on Robotics and Automation (2015) 3611 (DOI: 10.1109/ICRA.2015.7139700).

[13] A. Geiger, et al:: "Automatic camera and range sensor calibration using a single shot," IEEE International Conference on Robotics and Automation (2012) 3936 (DOI: 10.1109/ICRA.2012.6224570).

[14] Z. Liu, et al.: "An external parameter calibration method for multiple cameras based on laser rangefinder," Measurement 47 (2014) 954 (DOI: 10.1016/j.measurement.2013.10.029).

[15] V. D. Hoang, et al:: "Simple and efficient method for calibration of a camera and 2D laser rangefinder," Intelligent Information and Database Systems 8397 (2014) 561 (DOI: 10.1007/978-3-31905476-6_57).

[16] D. Scaramuzza, et al.: "Extrinsic self calibration of a camera and a 3D laser range finder from natural scenes," IEEE International Conference on Intelligent Robots and Systems (2007) 4164 (DOI: 10.1109/IROS.2007.4399276).

[17] Q. L. Zhang and R. Pless: "Extrinsic calibration of a camera and laser range finder (improves camera calibration)," IEEE International Conference on Intelligent Robots and Systems (2004) 2301 (DOI: 10.1109/IROS.2004.1389752).

[18] F. Vasconcelos, et al.: "A minimal solution for the extrinsic calibration of a camera and a laser-rangefinder," IEEE Trans. 
Pattern Anal. Mach. Intell. 34 (2012) 2097 (DOI: 10.1109/TPAMI. 2012.18).

[19] Z. W. Liu, et al.: "Extrinsic calibration of a single-point laser rangefinder and single camera," Opt. Quantum Electron. 51 (2019) 186 (DOI: 10.1007/s11082-019-1907-8).

[20] L. Liu, et al.: "A novel image analysis method for rotational motion of circular feature based on perspective projection," Imaging Sci. J. 63 (2015) 252 (DOI: 10.1179/1743131X15Y.0000000005).

[21] M. Z. Li, et al.: "Orbit determination for remote-sensing satellites using only optical imagery," Int. J. Remote Sens. 38 (2017) 1350 (DOI: 10.1080/01431161.2017.1280626).

[22] F. T. Yang, et al.: "A cost-effective non-orthogonal 3D measurement system," Measurement 128 (2018) 264 (DOI: 10.1016/ j.measurement.2018.06.048).

[23] J. H. Kang, et al.: "A novel calibration method of articulated laser sensor for trans-scale 3D measurement," Sensors 19 (2019) 1083 (DOI: 10.3390/s19051083).

[24] X. L. Wang, et al:: "Hardware implementation of fast and robust star centroid extraction with low resource cost," IEEE Sensors J. 15 (2015) 4857 (DOI: 10.1109/JSEN.2015.2428434).

[25] B. Gou, et al.: "Automatic centroid extraction method for noisy star image," IET Image Process. 12 (2018) 856 (DOI: 10.1049/iet-ipr. 2017.0979).

[26] L. Y. Luo, et al.: "Improved centroid extraction algorithm for autonomous star sensor," IET Image Process. 9 (2015) 901 (DOI: 10.1049/iet-ipr.2014.0488).

[27] F. Xu, et al.: "Modified Levenberg-Marquardt-based optimization method for LiDAR waveform decomposition," IEEE Geosci. Remote Sens. Lett. 13 (2016) 530 (DOI: 10.1109/LGRS.2016. 2522387).

[28] J. S. Smith, et al.: "Neural network training with LevenbergMarquardt and adaptable weight compression," IEEE Trans. Neural Netw. Learn. Syst. 30 (2019) 580 (DOI: 10.1109/TNNLS.2018. 2846775).

[29] C. Ma, et al:: "Globally convergent Levenberg-Marquardt method for phase retrieval,” IEEE Trans. Inf. Theory 65 (2019) 2343 (DOI: 10.1109/TIT.2018.2881187).

[30] J. Shawash, et al.: "Real-time nonlinear parameter estimation using the Levenberg-Marquardt algorithm on field programmable gate arrays," IEEE Trans. Ind. Electron. 60 (2013) 170 (DOI: 10.1109/ TIE.2012.2183833). 